\title{
Schema Therapy for Personality Disorders: a Qualitative Study of Patients' and Therapists' Perspectives
}

\author{
Noor de Klerk \\ NPI Specialist in Personality Pathology, the Netherlands
}

Tineke A. Abma

VU Medical Center, the Netherlands

Lotte L.M. Bamelis

Hospital Oost-Limburg, Belgium and Maastricht University, the Netherlands

Arnoud Arntz

University of Amsterdam, and Maastricht University, the Netherlands

\begin{abstract}
Background: Several studies have evaluated the (cost) effectiveness of schema therapy for personality disorders, but little research has been done on the perspectives of patients and therapists. Aim: The present study aims to explore patients' and therapists' perspectives on schema therapy. Method: Qualitative data were collected through in-depth semi-structured interviews with 15 patients and a focus group of 8 therapists. A thematic analysis was performed. Results: Most patients and therapists agreed that helpful aspects in schema therapy were the highly committed therapeutic relationship, the transparent and clear theoretical model, and the specific schema therapy techniques. About unhelpful aspects, several patients and some therapists shared the opinion that 50 sessions was not enough. Furthermore, patients lacked clear advance information about the possibility that they might temporarily experience stronger emotions during therapy and the possibility of having telephone contact outside session hours. They missed practical goals in the later stage of therapy. With regard to imagery, patients experienced time pressure and they missed a proper link between the past and the present. For therapists, it was hard to manage the therapeutic relation, to get used to a new kind of therapy and to keep the treatment focused on personality problems. Conclusions: Patients and therapists found some aspects of the schema therapy protocol helpful. Their views about which aspects are unhelpful and their recommendations need to be taken into consideration when adjusting the protocol and implementing schema therapy.
\end{abstract}

Keywords: Schema therapy, personality disorder, qualitative research, perspectives

Correspondence to Noor de Klerk, NPI Specialist in Personality Pathology, Amsterdam, the Netherlands. E-mail: noor.de.klerk@npsai.nl 


\section{Introduction}

Over the last decade, schema therapy (ST) has become an increasingly popular treatment model for patients with various mental health problems. ST was developed by Young in the 1980 s to treat patients with chronic personality problems who did not benefit sufficiently from traditional cognitive behavioural therapy. Elements of different therapeutic schools and (bio) psychological theories are integrated into a theoretical model based on cognitive therapy (Young, Klosko and Weishaar, 2003). A number of studies have demonstrated the (cost) effectiveness of ST, provided in an individual format, for borderline personality disorder (Giesen-Bloo et al., 2006; Nadort et al., 2009; Van Asselt et al., 2008) and other personality disorders (Bamelis, Evers, Spinhoven and Arntz, 2014; Bamelis, Arntz, Wetzelaer, Verdoorn and Evers, 2015; Gude and Hoffart, 2008; Gude, Monsen and Hoffart, 2001; Weertman and Arntz, 2007). More recently, schema therapy have been studied in group format and a combination of group and individual formats and showed promising results, both for borderline personality disorder (Dickhaut and Arntz, 2014; Farell, Shaw and Webber, 2009; Wetzelaer et al., 2014) and for other personality disorders (Renner et al., 2013; Skewes, Samson, Simpson and Van Vreeswijk, 2015).

However, little research has been done on the perspectives of patients and therapists with regard to ST. One study, by Spinhoven, Giesen-Bloo, Van Dyck, Kooiman and Arntz (2007), compared the quality of the therapeutic relationship in ST with that in transference-focused psychotherapy (TFP) in the case of treatment for borderline personality disorder. A qualitative study by Ten Napel-Schutz, Abma, Bamelis and Arntz (2011) focused on patients' perspective on imagery, an important technique specific to ST. Skewes et al. (2015) used feedback from patients in a pilot study on short-term group ST for mixed personality disorders. As far as we know, no study has yet been published that investigates the perspectives of both patients and therapists on a wide range of aspects of ST. The present study explores how patients and therapists experience ST, in order to develop ways to improve ST in the future.

\section{Method}

\section{Design}

This qualitative study was conducted on the basis of a multicentre, randomized and controlled trial (RCT) on the clinical (cost) effectiveness of individual ST with six personality disorders: avoidant, dependent, obsessive-compulsive, paranoid, histrionic and/or narcissistic personality disorder (Bamelis, Evers and Arntz, 2012; Bamelis et al., 2014, 2015). Approval was granted by the medical ethics committee of Maastricht University. Informed consent for participation in the semi-structured interviews was obtained from all patients.

\section{Sample}

Participants consisted of two groups: patients and therapists. Patients were recruited among the patients that underwent ST in the aforementioned RCT, with the exclusion of patients treated by the first author. "Theoretical sampling" and "variational sampling" (Barbour, 2001; Kuper, Lingard and Levinson, 2008) were used to select participants, both aimed at achieving a large variation. Theoretical sampling was employed by selecting both participants who had completed ST and participants who had prematurely ended ST (drop-outs). To conduct 
Table 1. Characteristics of interview patients (completers and drop-outs) and patients in the ST condition of the RCT

\begin{tabular}{llcc}
\hline & & $\begin{array}{l}\text { Interview } \\
\text { patients } \\
n=15\end{array}$ & $\begin{array}{l}\text { ST condition } \\
\text { RCT patients } \\
n=145\end{array}$ \\
\hline Age, mean $(S D)$ & & $34.27(8.56)$ & $37.57(9.69)$ \\
\hline Gender, nr (\%) & Male & $4(26.7)$ & $66(45.5)$ \\
Education, nr (\%) & Female & $11(73.3)$ & $79(55.5)$ \\
& Primary school & - & $6(4.1)$ \\
& Lower vocational & - & $6(4.1)$ \\
& Lower secondary & $1(6.7)$ & $22(15.2)$ \\
& Higher secondary & $2(13.3)$ & $11(7.6)$ \\
& Intermediate vocational & $5(33.3)$ & $46(31.7)$ \\
& Pre-university & $1(6.7)$ & $11(7.6)$ \\
& Higher vocational & $4(26.7)$ & $29(20)$ \\
& Academic & $2(13.3)$ & $14(9.7)$ \\
& House wife & - & $7(4.8)$ \\
& Student & $1(6.7)$ & $7(4.8)$ \\
& Employed & $9(60)$ & $66(45.5)$ \\
& Disability & $4(26.6)$ & $47(32.4)$ \\
& Welfare & $1(6.7)$ & $17(11.7)$ \\
& Retired & - & $1(0.7)$ \\
& Avoidant & $9(60)$ & $74(51)$ \\
& Dependent & - & $16(11)$ \\
& Obsessive compulsive & $3(20)$ & $41(28.3)$ \\
& Paranoid & $3(20)$ & $8(5.5)$ \\
& Narcissistic & - & $6(4.1)$ \\
\hline
\end{tabular}

variational sampling, patients who were treated by different therapists were selected from different mental health care institutes. Eleven of the patients who had completed ST were asked to participate and all agreed. Sampling of patients stopped when saturation of the emerging themes was reached (Green and Thorogood, 2004; Guest, Bunce and Johnson, 2012; Tong, Sainsbury and Craig, 2007). Furthermore, 14 drop-outs were approached, 8 of whom agreed to an interview. Two drop-outs turned out never to have started ST. One person dropped-out because she did not agree with the research protocol, more specifically with the recording of therapy sessions for study reasons, despite giving initial informed consent. One drop-out decided to continue another treatment she was already involved in. For this reason, the data of 4 drop-outs were included in the analysis. These dropouts stopped therapy at 2, 7, 15 and 40 sessions respectively; three of them for reasons concerning the content of ST and one involuntarily, due to practical reasons on the part of the mental health institute. Table 1 shows the characteristics of the 15 participating patients, including 4 drop-outs, compared with the complete ST condition of the RCT sample. Based on socio-demographic characteristics, the interviewees seemed fairly representative of the total ST condition. However, there are fewer diagnostic groups represented relative to the RCT. 
Table 2. Characteristics of focus group therapists and therapists in the ST condition of the RCT

\begin{tabular}{llcc}
\hline & & $\begin{array}{l}\text { Focus group } \\
\text { therapists } \\
n=8\end{array}$ & $\begin{array}{l}\text { ST condition } \\
\text { RCT therapists } \\
n=83\end{array}$ \\
\hline Age*, mean $(S D)$ & & $52(5.9)$ & $47.23(8.52)$ \\
\hline Gender, nr (\%) & Male & $2(25)$ & $30(36.1)$ \\
& Female & $6(75)$ & $53(63.9)$ \\
\hline Years of ST experience, mean $(S D)$ & & $3.75(1.39)$ & $2.2(1.81)$ \\
\hline Training cohort, nr (\%) & Lectures & $4(50)$ & $66(79.5)$ \\
Original frame of reference, $\mathrm{nr}(\%)$ & Experiential learning & $4(50)$ & $17(20.5)$ \\
& Cognitive behavioural therapy & $6(75)$ & $58(69.9)$ \\
& Client centred therapy & $2(25)$ & $9(10.8)$ \\
& Psychodynamic therapy & - & $3(3.6)$ \\
& Family therapy & - & $3(3.6)$ \\
& Group therapy & - & $2(2.4)$ \\
& Eclectic therapy & - & $3(3.6)$ \\
Patients treated by focus group, nr & Other & - & $5(6)$ \\
\hline Primary PD diagnosis, nr $(\%)$ & Avoidant & 20 & 145 \\
& Dependent & $11(55)$ & $74(51)$ \\
& Obsessive compulsive & $2(10)$ & $16(11)$ \\
& Paranoid & $5(25)$ & $41(28.3)$ \\
& Narcissistic & $1(5)$ & $8(5.5)$ \\
& & $1(5)$ & $6(4.2)$ \\
\hline
\end{tabular}

Participating therapists were recruited from the total group of therapists in the ST condition of the RCT. They were invited to register on a voluntary basis by mail. The criterion of "convenience sampling" was applied, as participants consisted solely of therapists who responded positively to the invitation (Kuper et al., 2008). Eight therapists, working in 4 different mental health institutes, took part. Some of the therapists were colleagues, others knew each other from the trial, and some therapists did not know each other. In total, the group of therapists had treated 20 patients. None of the therapists had treated any of the participating patients. Compared to the therapists in the ST condition of the RCT, therapists in the current study were of a higher average age, had more work experience, and were more experienced with ST. With regard to the diagnoses of the patients they treated, the participating therapists were representative of the larger group of therapists in the RCT (Table 2).

\section{Data collection}

In-depth semi-structured interviews were conducted with patients. The patients were questioned in an open and flexible manner about their experiences with, reflections on, and opinions about ST. The topics were based on questions that had arisen in the research group and were slightly adapted after three interviews. Prepared questions were used in every interview. The participants were also encouraged to discuss related topics that they considered relevant. 
The interviews were all conducted by the first author (NdK). She did not know any of the patients or their DSM-IV diagnoses in advance. All interviews were recorded using a digital recorder, transcribed and anonymized. There was no fixed time set for the interviews and they were ended when no new information emerged. The duration varied from 45 to 85 minutes with those who completed SFT (completers) and 10 to 20 minutes with dropouts. The interviews were held between October 2009 and September 2010. Interviewing took place at different mental health institutes and one interview was held at a participant's home. All interviews with drop-outs were held by telephone, as these participants were not willing to arrange an appointment in person. The data from therapists were collected by means of a focus group meeting, led by the first author. Prior to the meeting, three authors (NdK, TA and AA) drew up a number of generally phrased topics for discussion. During the focus group meeting, the therapists exchanged and discussed their experiences (Morgan, 1996). The session was recorded, transcribed and anonymized. The meeting took place in November 2009, lasted 90 minutes and was held at one of the participating mental health institutes

\section{Data analysis}

The first author (NdK) thematically analysed the data gathered in this study. The patient interviews and the results from the focus group were first analysed separately. NdK carefully read and re-read the transcripts of the interviews and made an analysis of the content. At this point the analysis was sent to the participants, who were asked whether they recognized themselves in the analysis and whether it clearly brought out what they had aimed to say. Twelve patients (eight completers and four dropouts) confirmed to have recognized themselves in the analysis, two respondents offered small corrections that were implemented, and one respondent did not respond to this "member check" request, despite several attempts to contact her. It was assumed that this participant agreed with the analysis. Subsequently, the first author (NdK) applied "open coding" by pinning labels (codes) on text fragments that seemed to form the basis of repeated patterns (themes) across the data set. The second author (TA) cross-checked the codes. Then NdK compared, combined and clustered all labels into potential overarching themes. Finally, NdK redefined themes by assessing them in relation to coded text fragments as well as to all the data. This way, it was ensured that the themes fully covered the pertinent text fragments. At this point, the themes extracted were again discussed with the second author (TA) until consensus was reached on their relevance. The same procedure was followed for the transcript of the focus group meeting (Braun and Clarke, 2006; Pope, Ziebland and Mays, 2000). With regard to the member check of therapists, seven therapists indicated they recognized themselves in the content. One therapist did not respond. Subsequently, responsive evaluation was used in order to gain further understanding of ST from a variety of perspectives (Abma, Nierse and Widdershoven, 2009; Stake, 2004). For this purpose, the themes from the interview and focus group transcripts were compared and similarities and differences were described (NdK and TA).

\section{Quality procedures}

Efforts were made in various ways to safeguard the internal validity. First, a "member check" was conducted (Green and Thorogood, 2004; Tong et al., 2007). Second, triangulation was used by obtaining data from patients and therapists (Barbour, 2001; Green and Thorogood, 2004; Tong et al., 2007). Furthermore, the internal validity and reliability were enhanced 
through "reflection". The researcher/first author kept a logbook of her experiences during the study, in order to be aware of her own "frames" and their potential influence. Moreover, the researcher consulted with the second author in two stages of the coding procedure, to prevent any distortions caused by personal and professional background (Green and Thorogood, 2004). In order to guarantee external validity, the researchers tried to give a clear description of the specific context of the study. Moreover, theoretical generalization has been made possible as themes were inductively generated from the data (Green and Thorogood, 2004).

\section{Results}

The results of the data analysis can be divided into three categories: aspects of ST that patients and therapists found helpful; aspects they found unhelpful; and recommendations for improving ST. Remarkably, patients and therapists strongly agreed on the helpful aspects. Some aspects were found unhelpful by both patients and therapists, others by either the patients or the therapists.

\section{Helpful aspects}

Therapeutic relationship. The therapeutic relationship in ST was regarded as positive, intensive and substantially different from other forms of psychotherapy by patients as well as therapists.

She kept pulling me. I could say: she was pressuring me, but I think that sounds negative, I feel more like she kept referring to my pitfalls. She persisted in mentioning those to get me to overcome them. (patient 5)

He took on the role of a father, of a kind of teacher, very pleasant. (patient 10)

The therapeutic relationship in ST is special, really different from other kinds of therapies, more involved. (therapist)

For some patients, an important element of the special therapeutic relationship was the possibility for short telephone contact with the therapist. The following quote concerns telephone contact outside the sessions.

It (contact outside the sessions) gives you the feeling that you can always get help from someone if you're having a hard time, that you always have someone to fall back on. Being aware that the option was there, did me so much good that I hardly needed to make use of it and when I did, just a brief conversation was sufficient. (patient 8)

Theoretical frame. The schema mode model, which is perceived by patients - and even by drop-outs - as a recognizable frame to better understand their own behaviours and feelings, was the second helpful aspect.

These modes - perhaps you can call them subpersonalities - these were very clear. The first time, she (the therapist) drew it for me on a flip-chart. I could still copy that now. I really took that in. Also after some time, I could easily do things with that myself. I noticed that I was also able to explain it properly to other people. (patient 11) 
Making my own defense mechanisms transparent, that protector that I developed, was useful. (drop-out patient 3)

Therapists believed the frame contributed to structuring the therapy.

It (the model) gives so much to hold on to and it is such a stepping stone. The whole therapy is on that table or on your lap, and we (patient and therapist) point at it. Where are we, what are we doing? You keep making the connection. (therapist)

\section{Specific ST techniques}

The techniques specific to ST, imagery in particular, were mentioned by both patients and therapists as a third helpful aspect.

That (imagery) evoked such intense emotions. I was crying my eyes out there and every time, it gave such a surprisingly huge feeling of relief. After the session I walked out as a new person and I saw things more positively and I instantly slept much better as well. (patient 5)

The intense emotions with imagery are necessary for change. (therapist)

The important shift from intellectually knowing to emotionally believing in the incorrectness of schemas, which ST exercises aim at, was phrased explicitly by a completer and a drop-out in the following two quotes:

You go back to a difficult situation in the past, but now there is someone else there who tells you at that moment that what is happening now, the way you are feeling, that this isn't wrong. That helps, as sometimes I get the idea that what happened back then had been my fault. Before, I might have able to put things into perspective, but that did not change the negative feelings. I found those exercises confrontational, but they helped to release me from that self-imposed miserable state of mind. (patient 4)

It's about letting go a little bit, what you have to do at such a moment ... loss of control ... emotionally surrendering to the situation. (drop-out patient 3 )

\section{Unhelpful aspects}

Therapeutic relationship. Although most patients perceived the therapeutic relation as an important helpful aspect of ST, for two patients the relationship with the therapist was unhelpful and even the reason to drop out.

The therapist was blaming my parents, which was very unsettling for me. For that reason, I immediately decided to stop therapy. (drop-out patient 1)

She was not very personal. She very much focused on the therapy itself ... In essence, I did sense that she wanted to support me. Only, I think she did not succeed in doing so. (drop-out patient 4)

At the same time, some therapists found the intensity and level of involvement of the therapeutic relationship to be highly demanding.

In the beginning you sigh sometimes, as if a new foster child is moving in with so many problems and it is so difficult, that you really need to sink your teeth into it again. (therapist) 
Furthermore, therapists experienced difficulties in familiarizing themselves with a new therapy. Some said they spent too much time thinking about the theory or found it hard to put their minds to doing exercises during the sessions, especially when they were not used to this in their original frame of reference.

There is a risk that you think too much because it's new and then you don't work instinctively enough. (therapist)

\section{Time pressure}

Several patients were of the opinion that 50 sessions is not enough to effect a substantial change.

Your twisted thinking patterns, they are so etched into your hard drive, it is so difficult to get rid of those, in order to get a normal pattern of thought. That will require more than 50 sessions. (patient 3)

Therapists had varying opinions about limiting therapy to 50 sessions. Some therapists felt this was not sufficient to realize significant improvement.

I often found it was too short and ended too abruptly. (therapist)

Other therapists considered it important to make clear in advance that at some point therapy will end.

You do not give the impression that forever remaining in therapy will solve everything. (therapist)

Patients also felt time pressure and too little rest with regards to imagery.

I put myself under pressure. Feeling as if we have all the time in the world, or that it doesn't have to be right now, that might have been reassuring. (patient 1)

\section{Lack of information}

For some patients it was unclear if there was a possibility to get in contact with the therapists by telephone outside the sessions.

Sometimes it was so intense and it made me think that something really needed to happen or else I would jump out of a window or whatever and then it was unclear whether I could call and I was too scared to do so as well. (patient 3)

Several patients suffered from very intense emotions during the process of gaining understanding and undergoing change brought on by therapy, which they had not been informed about in advance.

You go really deep and, initially, things went from bad to worse. I then needed reassurance and the people around me, who had experience with this said that things would get better. However, actually a therapist is the one who's supposed to say that. Maybe she tried to, but it just didn't register with me sufficiently. (patient 1) 


\section{Lack of practical application}

Some patients lacked clear practical targets, especially during the final phase of the treatment.

Insufficiently geared to practical situations, it remained theoretical, especially towards the end. ... I wanted to work more practically, try out things in the outside world. (patient 2)

Where imagery is concerned, several patients could not benefit when the connection between past and present was not properly made. In that case, it was not clear enough in what way imagery could contribute to actually solving their problems.

I specifically did not want to revisit the past because I thought: "I am in the present". Going back to the past to me seemed like something that didn't have much to do with the current situation, whereas it was an enormous burden for me. (patient 6)

\section{No shared focus between patient and therapist}

In some cases, therapists struggled to perform schema therapy because patients were not "ready" to focus on the personality problems. The reasons for this, for instance, were that there was too much agitation in their lives at the time, that patients did not link their problems to their personalities yet or that there was too much avoidance.

One client who was involved in a fierce divorce battle, literally said to me that he could not afford to be unstable at that time and I seriously considered to stop ST, at least temporarily. (therapist)

It must be possible to explicitly formulate the conditions for treatment focusing on personality disorder, otherwise ST will fail. (therapist)

\section{Recommendations}

Both patients and therapists gave recommendations on how to improve the effectiveness of ST. Some of these suggestions arose as a corollary of the unhelpful aspects referred to earlier.

Length, supportive and motivational elements. Patients recommended a therapy of over 50 sessions and one patient suggested there should be a possibility for evening sessions for patients who work. Therapists suggested allowing for more flexibility in length, and adding supporting and motivational techniques for patients currently in difficult circumstances or patients who are not yet ready for a change of personality problems because of avoidance.

I consider the 50 sessions more of a guideline. You can have a patient come back a number of times after a year if this is necessary, precisely because of reparenting. After all, when your children move out, you don't tell them goodbye and to never come back. (therapist)

I believe that as ST therapists we should also feel at liberty to add elements from other therapies if necessary, for example more supporting techniques or starting a motivational course. (therapist)

Clear information in advance. Patients also felt that information about the treatment should be presented more clearly in advance, for example with regard to the possibility that they might 
experience stronger emotions during treatment or the possibility to contact their therapist by telephone outside the sessions.

Proper connection between the present and the past in imagery. Where imagery is concerned, the importance of the connection between the present and the past is emphasized.

You do need a session to properly work out the imagery in the here and now - what can you do with it, what purpose does it serve? - in order to make a good connection between past and present. (therapist)

More responsibility for patients in the final phase of the therapy. Therapists emphasized the importance of placing the responsibility for recovery more on the patient in the final phase of the therapy. Recommendations to achieve this were: asking patients to mail an agenda for the session prior to it being held, agreeing very specifically on targets and having patients monitor the progress of the targets.

She (the patient) must choose how she is going to do this, perhaps then she will also anticipate the following steps. Otherwise, as a therapist, you will be continuously pulling the cart. In my opinion a shift should occur in that respect, towards the end of the therapy. (therapist)

Experiential training for therapists. Therapists suggested that a training program with a focus on experiential learning, using role plays and individual feedback, is important to make therapists feel fully equipped to work with schema therapy. That may reduce the inconvenience of a new reference framework for therapists.

This is also an "active therapy", so in order to really learn this you need to assume an active role in the course as well. (therapist)

For an overview of helpful and unhelpful aspects, and recommendations made by patients and therapists, see Table 3.

\section{Discussion}

\section{Findings in the context of previous literature}

The overall high appreciation of the therapeutic relationship in ST is in line with previous literature (Spinhoven et al., 2007). Independent raters confirmed the high quality of the therapeutic relationship in ST by judging facilitative conditions (including empathy) to be higher in ST than in usual treatment and clarification-oriented psychotherapy (Bamelis et al., 2014). Moreover, the theoretical framework as an important mechanism was found previously in an open study that demonstrated that modification of early maladaptive schemas contributes to resolving symptomatic distress (Nordahl, Holthe and Haugum, 2005). These results suggest that many patients and therapists consider the schema mode model to be a valuable framework for understanding the patient's problems. This supports the current tendency in ST to base treatment of severe chronic problems on the basis of the schema mode model. Remarkably, patients in the current study did not report finding the focus of ST too narrow. This is contrary to the results of a qualitative study on patients' perspectives on Mentalization-Based Therapy (MBT) and Dialectical Behavioural Therapy (DBT), both of which were found to focus only on one main area and therefore did not address all targets that were important to patients (Katsakou et al., 2012). Although diagnostic categories differ between the studies, 
Table 3. Helpful aspects, unhelpful aspects and recommendations from patients and therapists

\begin{tabular}{|c|c|c|}
\hline & Patients & Therapists \\
\hline Helpful aspects & \multicolumn{2}{|c|}{$\begin{array}{l}\text { Therapeutic relationship } \\
\text { Theoretical frame } \\
\text { Specific ST techniques }\end{array}$} \\
\hline \multirow[t]{6}{*}{ Unhelpful aspects } & \multicolumn{2}{|c|}{50 sessions not enough } \\
\hline & $\begin{array}{l}\text { Lack of information about the } \\
\text { possibility of telephone } \\
\text { contact outside sessions }\end{array}$ & $\begin{array}{l}\text { Hard and taxing therapeutic } \\
\text { relationship }\end{array}$ \\
\hline & $\begin{array}{l}\text { Lack of information about the } \\
\text { possibility of strong emotions }\end{array}$ & $\begin{array}{l}\text { Getting familiar with a new } \\
\text { therapy }\end{array}$ \\
\hline & $\begin{array}{l}\text { Lack of concrete targets in the } \\
\text { final phase }\end{array}$ & $\begin{array}{l}\text { No shared focus on personality } \\
\text { problems }\end{array}$ \\
\hline & $\begin{array}{l}\text { Connection between the present } \\
\text { and the past within imagery } \\
\text { not enough clear }\end{array}$ & \\
\hline & $\begin{array}{l}\text { Too much time-pressure within } \\
\text { imagery }\end{array}$ & \\
\hline \multirow[t]{5}{*}{ Recommendations } & Possibility of more sessions & Flexibility in number of sessions \\
\hline & Clear information in advance & $\begin{array}{l}\text { Add supporting or motivational } \\
\text { techniques }\end{array}$ \\
\hline & & $\begin{array}{l}\text { Proper connection between the } \\
\text { present and the past within } \\
\text { imagery }\end{array}$ \\
\hline & & $\begin{array}{l}\text { Responsibility for patients in the } \\
\text { final phase }\end{array}$ \\
\hline & & $\begin{array}{l}\text { Experience-aimed training for } \\
\text { therapists }\end{array}$ \\
\hline
\end{tabular}

our findings support previous suggestions that ST might have a broader focus than many other approaches for treatment of personality disorders and thus meets more needs of patients. The last mechanisms referred to were specific ST techniques. Imagery has previously proved to be effective in treatment of Axis-II disorders (Arntz, 2011; Weertman and Arntz, 2007) but is at least initially experienced by many patients as confrontational (Ten Napel- Schutz et al., 2011). More specifically, suggestions to provide a clear structure with regard to imagery and to allow for sufficient time and an absence of pressure had been made earlier by patients in qualitative research (Ten Napel-Schutz et al., 2011). The importance of a proper connection between the present and the past had been stated before by Young et al. (2003).

This study identified a number of important aspects that had a hampering effect on ST, and several suggestions were made to solve them. Although the therapeutic relation was seen as an important working mechanism, therapists found it hard and taxing. To allow therapists to function optimally with this difficult patient population, support in the form of supervision, peer discussion and experience-aimed training seems essential. The problem with therapists' unfamiliarity with the new therapeutic framework supports the findings from Bamelis et al. (2014) that more therapeutic experience enhances ST outcome, and is in line with previous research on the influence of therapist experience and training (Stein and Lambert, 1995; 
Weertman and Arntz, 2007). Therapists recommend providing an active ST training course, enabling them to gain experience at an early stage. The influence of training method on outcome had already become apparent from Bamelis et al. (2014) and Sholomskas et al. (2005). While patients thought the theoretical model was clear, they lacked clear information concerning the possibility to have telephone contact outside the sessions. If telephone contact was made available, some patients felt no reason to use it. However, some patients certainly used telephone contact and claimed to benefit from it. For other patients the fact that they had this option caused such a supportive feeling that they did not need to use it at all, or only very infrequently for a short conversation. This is partly in contrast to Nadort et al. (2009), who found that telephone crisis support outside office hours did not affect the outcome. However, the other treatment condition in the study by Nadort et al. (2009) allowed for telephone contact during office hours, and this might be enough for most patients. Furthermore, e-mail might replace telephone contact, reducing the burden for therapists whilst offering the patient a connection to the therapist. Patients also felt they were not sufficiently informed in advance regarding the fact that they might experience stronger emotions during therapy. Ten NapelSchutz et al. (2011) found that patients misunderstood the information they were presented with in relation to the effects of imagery. It seems that patients with personality problems need to be presented with more comprehensive information and to be given this information repeatedly, especially if this information relates to possible fears they may have. In treating patients for whom a focus on changing personality problems is not yet possible, therapists' suggestion to allow for more flexibility in the protocol with regard to the number of sessions and to include supporting and motivational techniques may be helpful. This suggestion comes besides the advice by Bamelis et al. (2014) to perform an assessment on readiness to change before the start of treatment, offer a motivation-enhancing module if indicated, and to sharpen the indication criteria.

An interesting finding in this study was the connection several patients and some therapists made between limiting therapy to 50 sessions and the insufficient improvement among some patients. This opinion reflects the clinical view that realizing a change in personality problems is very difficult and time-consuming. However, since Bamelis et al. (2014) found major improvement with 50 sessions, including ongoing progress in the second year (only 10 sessions) and third year (no sessions), it is questionable whether limited treatment duration was the cause of little progress. In order to enhance outcome, we should not only consider more flexibility in length of treatment, but we could also follow another suggestion that arose from this study, which is giving patients more responsibility in the final phase, allowing them to take control of their lives (Arntz, 2012). In this context, patients' comment regarding the lack of concrete targets in the final phase should be taken seriously, as these concrete targets may help to assume this necessary responsibility. After all, unlimited treatment would imply higher costs and might cause a delay in working on issues during therapy.

\section{Strengths and limitations}

To our knowledge, this is the first qualitative study exploring and comparing both patients' and therapists' experiences with ST. Nevertheless, one still needs to be careful when generalizing the results to other patients and ST therapists. The results were based on a relatively small group of patients and therapists. Also, despite the fact that the groups were quite representative of the samples of the trial, there were some differences. In the patient group, not all diagnostic 
groups were represented, which may have affected results. On average, the therapists were more experienced, so they presumably faced fewer problems with ST. Furthermore, opinions of patients may have been coloured by feasibility and their willingness to participate in research, and also by unresolved personality problems. Data from drop-out patients were limited, because many refused the interview and those that consented were not willing to engage in a long conversation. Moreover, the study was conducted by a researcher who practices schema therapy herself, which enabled her to properly understand the subject matter. However, this might also have had an impact on the process of data collection and analysis, although a member-check was done and the analyses were double checked by the second author. Lastly, there was no full guarantee of the validity of the labels, although a member check was performed, because part of the process of determining labels took place after this check.

\section{Acknowledgements}

Experiments conceived and designed by: NdK, TA, LB. Experiments performed by: NdK. Data analysis: NdK, TA. Reagents/materials/analysis tools contributed by: NdK, TA. Paper written by: NdK. Feedback to the manuscript: TA, LB, AA.

Financial support: This research is funded by grant 945-06-406 from ZonMW, the Netherlands Organization for Health Research and Development, awarded to Arnoud Arntz. Funders had no role in study design; in collection, analysis and interpretation of data; in writing the report; or in the decision to submit the article for publication. Researchers acted independently from funding sources.

Conflict of interest: The authors have no conflicts of interest with respect to this publication.

\section{References}

Abma, T. A., Nierse, C. J. and Widdershoven, G. A. M. (2009). Patients as partners in responsive research: methodological notions for collaborations in mixed research teams. Qualitative Health Research, 19, 401-4015. doi:10.1177/1049732309331869

Arntz, A. (2011). Imagery rescripting for personality disorders. Cognitive and Behavioral Practice, 18, 466-481. doi:10.1016/j.cbpra.2011.04.006

Arntz, A. (2012). Schema therapy for cluster C personality disorders. In M. van Vreeswijk, J. Broersen and M. Nadort (Eds.), The Wiley-Blackwell Handbook of Schema Therapy: theory, research and practice (pp. 397-414). Chichester: Wiley- Blackwell.

Bamelis, L. L. M., Evers, S. M. A. A. and Arntz, A. (2012). Design of a multicentered randomized controlled trial on the clinical and cost effectiveness of schema therapy for personality disorders. BMC Public Health, 12, 75. doi:10.1186/1471-2458-12-75

Bamelis, L. L. M., Evers, S. M. A. A., Spinhoven, P. and Arntz, A. (2014). Results of a multicenter randomized controlled trial of the clinical effectiveness of schema therapy for personality disorders. The American Journal of Psychiatry, 171, 305-322. doi:10.1176/appi.ajp.2013.12040518

Bamelis, L. L. M., Arntz, A., Wetzelaer, P., Verdoorn, R. and Evers, S. M. A. A. (2015). Economic evaluation of schema therapy and clarification-oriented psychotherapy for personality disorders: a multicenter, randomized controlled trial. Journal of Clinical Psychiatry, 76, e1432-e1440. doi: 10.4088/JCP.14m09412

Barbour, R. (2001). Checklists for improving rigour in qualitative research: a case of the tail wagging the dog? British Medical Journal, 322, 1115-1117. doi: 10.1136/bmj.322.7294.1115 
Braun, V. and Clarke, V. (2006). Using thematic analysis in psychology. Qualitative Research in Psychology, 3, 77-101. doi:10.1191/1478088706qp063oa

Dickhaut, V. and Arntz, A. (2014). Combined group and individual schema therapy for borderline personality disorder: a pilot study. Journal of Behavior Therapy and Experimental Psychiatry, 45, 242-251. doi:10.1016/j.jbtep.2013.11.004

Farrell, J. M., Shaw, I. A. and Webber, M. A. (2009). A schema-focused approach to group psychotherapy for outpatients with borderline personality disorder: a randomized controlled trial. Journal of Behavior Therapy and Experimental Psychiatry, 40, 317-328. doi:10.1016/j.jbtep.2009.01.002

Giesen- Bloo, J., van Dyck, R., Spinhoven, P., van Tilburg, W., Dirksen, C., van Asselt, T., et al. (2006). Outpatient psychotherapy for borderline personality disorder: randomized trial of schemafocused therapy vs transference-focused psychotherapy. Archives of General Psychiatry, 63, 649658. doi:10.1001/archpsyc.63.6.649

Green, J. and Thorogood, N. (2004). Qualitative Methods for Health Research. London: Sage.

Gude, T. and Hoffart, A. (2008). Change in interpersonal problems after cognitive agoraphobia and schema-focused therapy versus psychodynamic treatment as usual of inpatients with agoraphobia and cluster C personality disorders: health and disability. Scandinavian Journal of Psychology, 49, 195-199. doi:10.1111/j.1467-9450.2008.00629.x

Gude, T., Monsen, J. T. and Hoffart, A. (2001). Schemas, affect consciousness, and cluster C personality pathology: a prospective one-year follow-up study of patients in a schema-focused shortterm treatment program. Psychotherapy Research, 11, 85-98. doi:10.1093/ptr/11.1.85

Guest, G., Bunce, A. and Johnson, L. (2012). How many interviews are enough? An experiment with data saturation and variability. Field Methods, 18, 59-82. doi:10.1177/1525822X05279903

Katsakou, C., Marougka, S., Barnicot, K., Savill, M., White, H., Lockwood, K., et al. (2012). Recovery in borderline personality disorder (BPD): a qualitative study of services users' perspectives. PLoS ONE, 7, e36517. doi:10.1371/journal.pone.0036517

Kuper, A., Lingard, L. and Levinson, W. (2008). Critically appraising qualitative research. British Medical Journal, 337, 687-692. doi:10.1136/bmj.a1035

Morgan, D.L. (1996). Focus groups. Annual Review Sociology, 22, 129-152. doi:10.1146/annurev.soc.22.1.129

Nadort, M., Arntz, A., Smit, J. H., Giesen- Bloo, J., Eikelenboom, M., Spinhoven, P., et al. (2009). Implementation of outpatient schema therapy for borderline personality disorder with versus without crisis support by the therapist outside office hours: a randomized trial. Behaviour Research and Therapy, 47, 961-973. doi:10.1016/j.brat.2009.07.013

Nordahl, H. M., Holthe, H. and Haugum, J. A. (2005). Early maladaptive schemas in patients with or without personality disorders: does schema modification predict symptomatic relief? Clinical Psychology and Psychotherapy, 12, 142-149. doi:10.1002/cpp.430

Pope, C., Ziebland, S. and Mays, N. (2000). Qualitative research in health care: analysing qualitative data. British Medical Journal, 320, 114-116. doi:10.1136/bmj.320.7227.114

Renner, F., Van Goor, M., Huibers, M., Arntz, A., Butz, B. and Bernstein, D. (2013). Short-term group schema cognitive-behavioral therapy for young adults with personality disorders and personality disorder features: associations with changes in symptomatic distress, schemas, schema modes and coping styles. Behaviour Research and Therapy, 51, 487-492. doi:10.1016/j.brat.2013.05.011

Sholomskas, D. E., Syracuse-Siewert, G., Rounsaville, B. J., Ball, S. A., Nuro, K. F. and Carroll, K. M. (2005). We don't train in vain: a dissemination trial of three strategies of training clinicians in cognitive-behavioral therapy. Journal of Consulting and Clinical Psychology, 73, 106-115. doi:10.1037/0022-006X.73.1.106

Skewes, S. A., Samson, R.A., Simpson, S.G. and van Vreeswijk, M. (2015). Short-term group schema therapy for mixed personality disorders: a pilot study. Frontiers in Psychology, 22, 1592. doi:10.3389/fpsyg.2014.01592 
Spinhoven, P., Giesen-Bloo, J. H., van Dyck, R., Kooiman, K. and Arntz, A. (2007). The therapeutic alliance in schema-focused therapy and transference-focused psychotherapy for borderline personality disorder. Journal of Consulting and Clinical Psychology, 75, 104-115. doi:10.1037/0022-006X.75.1.104

Stake, R. (2004). Standards-based and Responsive Evaluation. London: Sage.

Stein, D. M. and Lambert, M. J. (1995). Graduate training in psychotherapy: are therapy outcomes enhanced? Journal of Consulting and Clinical Psychology, 63, 182-196. doi: 10.1037/0022-006X.63.2.182

Ten Napel-Schutz, M. C., Abma, T. A., Bamelis, L. and Arntz, A. (2011). Personality disorder patients' perspectives on the introduction of imagery within schema therapy: a qualitative study of patients' experiences. Cognitive and Behavioral Practice, 18, 482-490. doi:10.1016/j.cbpra.2011.04.005

Tong, A., Sainsbury, P. and Craig, J. (2007). Consolidated criteria for reporting qualitative research (COREQ): a 32-item checklist for interviews and focus groups. International Journal of Quality in Health Care, 19, 349-357. doi:10.1093/intqhc/mzm042

Van Asselt, A.D.I., Dirksen, C.D., Arntz, A., Giesen- Bloo, J.H., van Dyck, R., Spinhoven, P., et al. (2008). Outpatient psychotherapy for borderline personality disorder: cost-effectiveness of schemafocused therapy versus transference focused psychotherapy. British Journal of Psychiatry, 192, 450457. doi:10.1192/bjp.bp.106.033597

Weertman, A. and Arntz, A. (2007). Effectiveness of treatment of childhood memories in cognitive therapy for personality disorders: a controlled study contrasting methods focusing on the present and methods focusing on childhood memories. Behaviour Research and Therapy, 45, 2133-2143. doi:10.1016/j.brat.2007.02.013

Wetzelaer, P., Farrell, J., Evers, S.M.A.A., Jacob, G., Lee, C.W., Brand, O., et al. (2014). Design of an international multicentre RCT on group schema therapy for borderline personality disorder. $B M C$ Psychiatry. 14, 319. doi:10.1186/s12888-014-0319-3

Young, J. E., Klosko, J. S. and Weishaar, M.E. (2003). Schema Therapy: a practitioner's guide. New York: Guilford Press. 